\title{
OPEN New cine magnetic resonance imaging parameters for the differential diagnosis of chronic intestinal pseudo-obstruction
}

\author{
Hiroki Sato ${ }^{1 凶}$, Hiroyuki Ogihara ${ }^{2}$, Kazuya Takahashi $^{1}$, Yuzo Kawata ${ }^{1}$, Yuichi Kojima $^{1}$, \\ Kentaro Tominaga ${ }^{1}$, Junji Yokoyama ${ }^{1}$, Yoshihiko Hamamoto ${ }^{2} \&$ Shuji Terai $^{1}$
}

Chronic intestinal pseudo-obstruction (CIPO) is a severe and refractory intestinal motility disorder whose diagnosis currently relies on subjective imaging assessments. Cine magnetic resonance imaging (MRI) may potentially improve the quantitative analysis of gastrointestinal motility; however, suitable CIPO detection parameters should be determined. Cine MRI was performed in seven patients with CIPO and 11 healthy controls. The logarithm of the Mahalanobis distance $\left(x_{1}\right)$ and distance variation per time $\left(\mathrm{x}_{2}\right)$ were used as the original parameters to determine CIPO diagnostic thresholds. Furthermore, the correlation between cine MRI findings and CIPO severity was investigated. Threshold values of $\alpha=1.10$ and $\beta=0.15$ for $x_{1}$ and $x_{2}$, respectively, produced a CIPO diagnosis sensitivity of $1.00(7 / 7)$ and specificity of $0.82(9 / 11)$. The resulting error was $0.11(2 / 18)$. The two parameters were correlated (Pearson's correlation coefficient: -0.52 ). Any of the intestinal tracts of patients with severe CIPO requiring home parenteral nutrition belonged to the region defined by $x_{1} \geq 1.10$ and $x_{2} \leq 0.15$. Cine MRI is effective for the quantitative evaluation of small intestinal motility and CIPO diagnosis when using the abovementioned parameters and can be useful for treatment decision-making. However, these parameters have a wide distribution in healthy volunteers; this may complicate the detection of other disorders.

Chronic intestinal pseudo-obstruction (CIPO) is a rare refractory disorder of the intestine ${ }^{1}$ wherein intestinal motility is severely impaired. It causes patients to experience abdominal pain, distention, vomiting, and weight loss despite the absence of organic disorders in the intestinal lumen. Recurrent paralytic ileus is a complication that causes social discomfort and necessitates repeated hospitalization for intestinal tract decompression or (at least) bowel rest. Furthermore, intestinal strangulation requires surgical resection of the necrotic segment, as that is the only strategy that can save the patient's life. However, patients who require resection of the intestine risk developing adhesive ileus or short bowel syndrome when extensive resection of the small intestine is performed. If their symptoms become more severe owing to intestinal motility failure, or malabsorption occurs owing to short bowel syndrome, home parenteral nutrition (HPN) becomes necessary to supplement nourishment ${ }^{2}$.

Histological examination of the impaired intestine is a common approach to investigate CIPO of unknown etiology. Based on previously reported patients, CIPO is considered a collection of several pathologies that include neuropathy, abnormalities of intestinal cells of Cajal, and myopathies ${ }^{3-6}$. However, in most cases, histological investigation does not yield a diagnosis, suggesting that CIPO has molecularly heterogeneous underlying causes. Furthermore, researchers are able to obtain specimens only from patients with advanced disease given that obtaining samples for histology will not influence treatment decision-making, and such procedures may worsen the underlying dysmotility (as mentioned above).

Histological investigation can only be performed for critical patients and the subsequent clinical course does not necessarily improve (and can even worsen in some cases); therefore, radiological imaging is preferable for

${ }^{1}$ Division of Gastroenterology, Graduate School of Medical and Dental Sciences, Niigata University, 757-1, Asahimachidori, Chuo-ku, Niigata-city, Niigata 951-8510, Japan. 2Division of Electrical, Electronic, and Information Engineering, Yamaguchi University Graduate School of Sciences and Technology for Innovation, Yamaguchi 755-8611, Japan. ${ }^{\square}$ email: pyloki-sato@med.niigata-u.ac.jp 


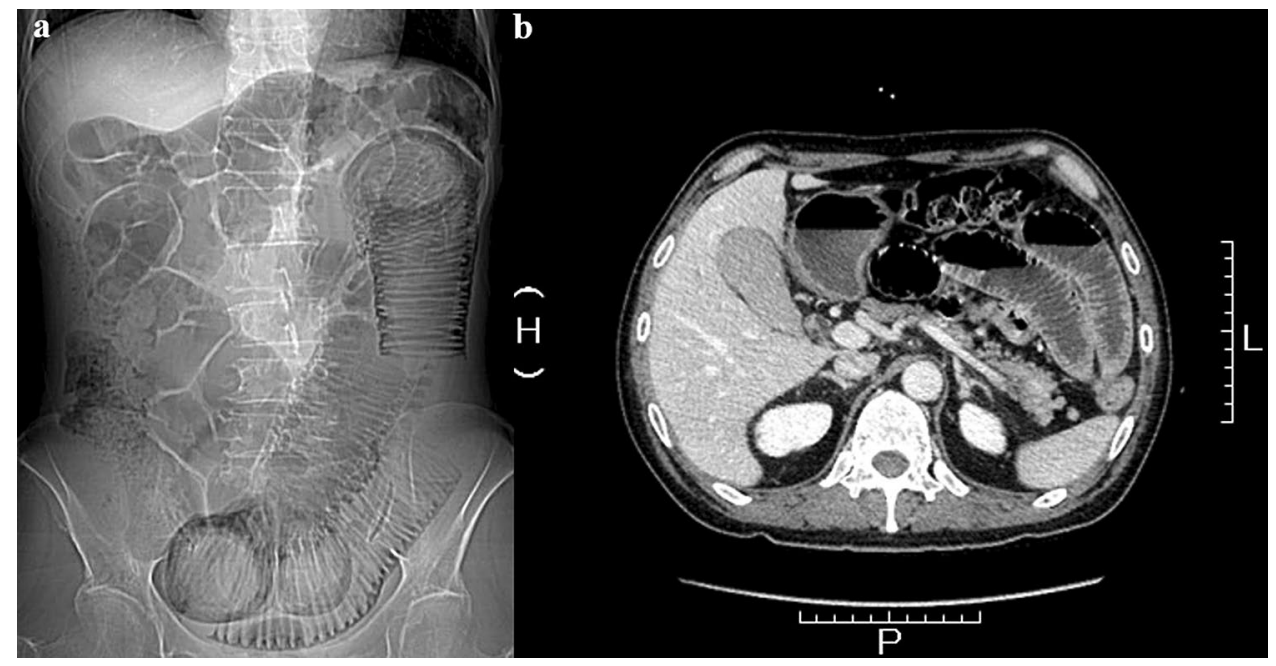

Figure 1. Typical radiological images of chronic intestinal pseudo-obstruction including plain radiograph (a) and computed tomography (b).

diagnosing CIPO. In typical cases, non-obstructive ileus findings are observed on radiography and computed tomography (Fig. 1). However, these modalities carry a risk of radiation exposure; moreover, radiography is not sufficiently sensitive or specific to detect ileus, particularly in its early stages when symptoms are relatively light and/or the duration since disease onset has been relatively short ${ }^{1}$. Therefore, other medical methodologies for the quantitative analysis of intestinal motility are recommended for CIPO diagnosis. Several modalities have been reported to be suitable for this purpose, although none are considered a gold standard owing to varying advantages and disadvantages ${ }^{7-9}$. For example, intestinal manometry can analyze the peristaltic wave at a certain point; however, the limited length of the catheter makes it impossible to analyze the distal parts of the intestine. Small bowel transit time on capsule endoscopy has intra-and inter-individual discrepancies ${ }^{10}$, and capsule endoscopy carries the risk of retention and is contraindicated in patients with CIPO. On the other hand, recent advances in magnetic resonance imaging (MRI) have led to the development of cine MRI, a noninvasive radiation-free tool to assess organ function and diagnose certain diseases, particularly for cardiovascular conditions ${ }^{11}$. As such, cine MRI can be applicable to gastrointestinal disorders, including $\mathrm{CIPO}^{12,13}$, and to establish parameters for cine $\mathrm{MRI}$ readings is an unmet requirement in $\mathrm{CIPO}^{14}$. Moreover, the inter- and intra-observer discrepancy remains a hindrance for objective diagnosis.

Therefore, the aim of this study was to establish new parameters for cine MRI that would assist in the diagnosis of CIPO.

\section{Methods}

Cine MRI protocol. CIPO was diagnosed in cases having long-term persistent abdominal symptoms for more than 6 months, no mechanical cause, bowel dilatation with air-fluid levels in radiological images ${ }^{1}$. After $8 \mathrm{~h}$ of fasting, the participants underwent MRI in the supine position after orally ingesting $1000 \mathrm{~mL}$ of water to fill the small intestine. Patients who were unable to ingest the entire amount because of severe abdominal symptoms were encouraged to drink as much as possible to prevent worsening of symptoms ${ }^{13}$.

Imaging was performed using a 1.5-T MRI unit (SIGNA ${ }^{\mathrm{Tx}}$ Creator, GE Healthcare, USA). Before performing cine MRI, coronal images of the entire abdomen were obtained to identify an appropriate imaging plane. A steady-state free precession sequence (FIESTA sequence: repetition time $=3.6 \mathrm{~ms}$, echo time $=1.6 \mathrm{~ms}$, flip angle $=70^{\circ}$, slice thickness $=10 \mathrm{~mm}$, matrix $=196 \times 230$, field of view $=400 \times 400 \mathrm{~mm}$, number of excitations $=1.0$, bandwidth $=100 \mathrm{kHz}$, and fat suppression = special [inversion time $=200 \mathrm{~ms}$ ]) was used for imaging, which allowed for continuous scanning without intervals for each image. Three slice location were separately acquired. For each slice location, sequential scanning was performed every $0.571 \mathrm{~s}$ with 35 images. The patients held their breaths for $20 \mathrm{~s}$.

All procedures involving human participants were performed in accordance with the principles of the 1964 Declaration of Helsinki. Niigata university hospital review board approval was obtained (approval no: 2018-0403) and written informed consent was obtained from all patients.

Data analysis. Cine MRI imaging analysis was performed using the Centricity DICOM Viewer software v. 2.2 (GE Medical Systems). An unsharp masking filter was used to improve the quality of all cine MRI scans degraded by noise (Fig. 2$)^{15}$.

First, the observer marked the intestinal tracts, which sequentially appeared in all the 35 MRIs, and then visually selected a set of corresponding intestinal tracts from each of the $35 \mathrm{MRI}$ images. The process of extracting a two-dimensional feature vector from the intestinal tract of interest for a patient with CIPO in MRI images is shown in Fig. 3. The intestinal tract of interest was represented as a two-dimensional feature vector and plotted 


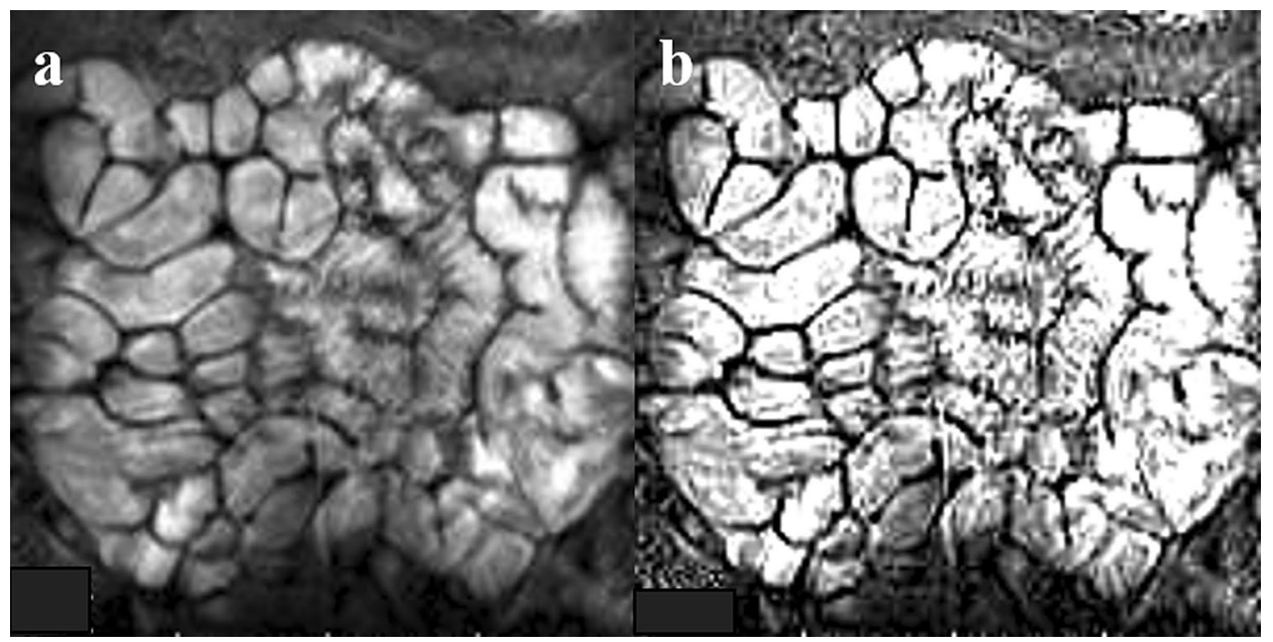

Figure 2. Edge of the small intestine can be enhanced automatically using software. Shown are (a) before enhancement and (b) afterward.

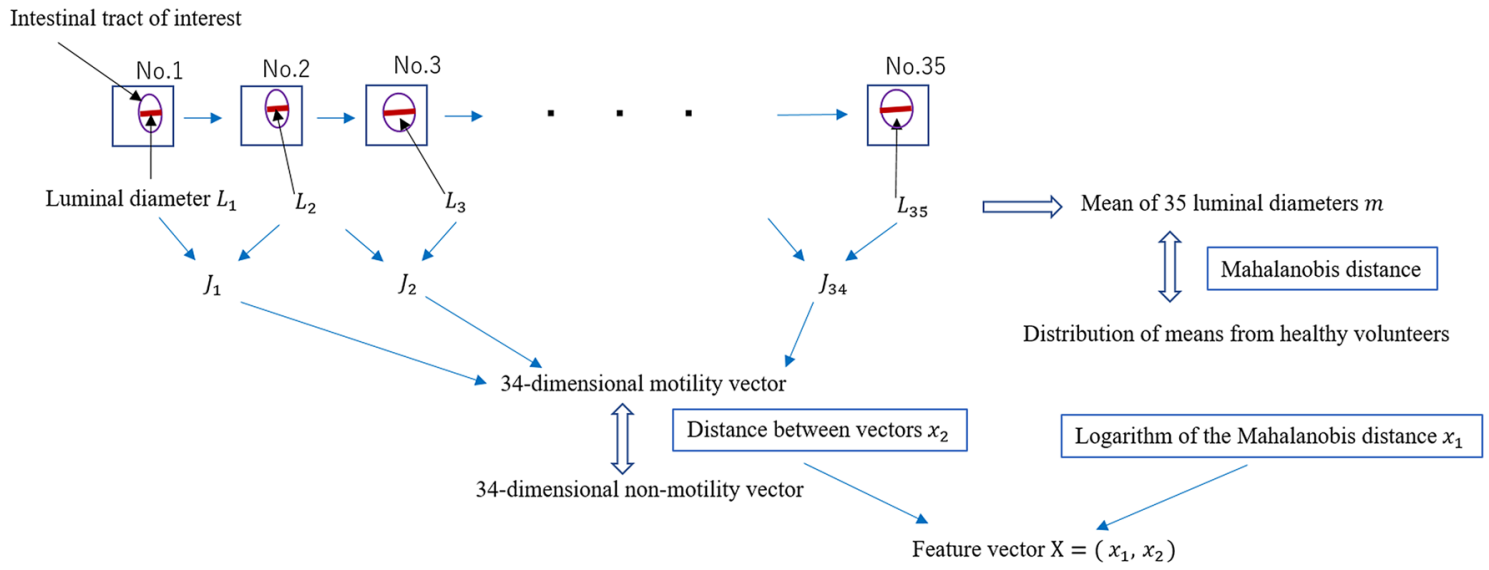

Extraction of a feature vector $\mathrm{X}=\left(x_{1}, x_{2}\right)$ from an intestinal tract for a patient with CIPO

Figure 3. Process of extracting a two-dimensional feature vector from an intestinal tract of interest for a patient with chronic intestinal pseudo-obstruction in magnetic resonance imaging.

on the two-dimensional feature space. We examined 78 intestinal tracts of interest from 18 samples. For each of the intestinal tracts of interest, 35 luminal diameters were separately measured. The total number of luminal diameters measured, which were used to calculate the J values and Mahalanobis distance, was 2730. With this method, it is important to consider inter-observer variability, because the variability is mainly produced by the observer. Hence, each luminal diameter was independently measured by two observers. Next, we checked the differences in the measurements performed by both observers and repeated the luminal diameter measurement in cases where a large difference was observed. The mean $m$ of the 35 luminal diameters was estimated. Similarly, the means from each of the healthy volunteers and the mean $\mu$ and variance $\sigma^{2}$ of the resulting means were also estimated. Next, the Mahalanobis distance was calculated, and its logarithm was obtained as the first feature $\mathrm{x}_{1}$. On the other hand, J values were calculated from luminal diameters, and the 34-dimensional motility vector was generated using J values. Then, the distance between the two vectors was adopted as the second feature, i.e., distance variation per time $\mathrm{x}_{2}$. The method is described in detail below.

At the corresponding location of the small intestinal tract, a line perpendicular to the long axis was drawn; this task was repeated for all sequential cine MRIs (Fig. 4). Using these diameters measured on 35 cine MRIs, the criterion $\mathrm{J}_{\mathrm{t}}$, which evaluates the motility of an intestinal tract, was defined by

$$
\mathrm{J}_{\mathrm{t}}=\frac{\mathrm{L}_{\mathrm{t}+1}}{\mathrm{~L}_{\mathrm{t}}+\mathrm{L}_{\mathrm{t}+1}} t=1,2, \ldots, 34
$$




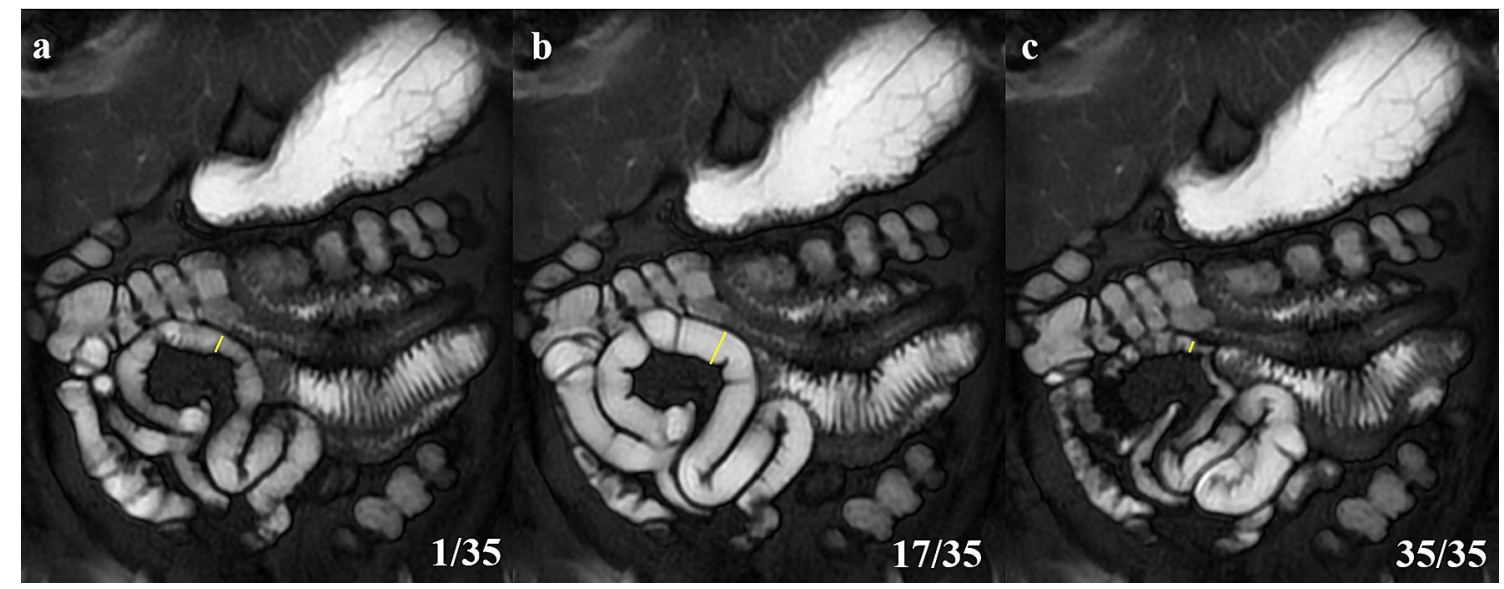

Figure 4. Luminal diameters of the small intestine (yellow line) are calculated using the software. This task was repeated for all sequential cine magnetic resonance imaging. The typical findings are shown as (a) (1st of the series), (b) (17th of the series), and (c) (35th of the series).
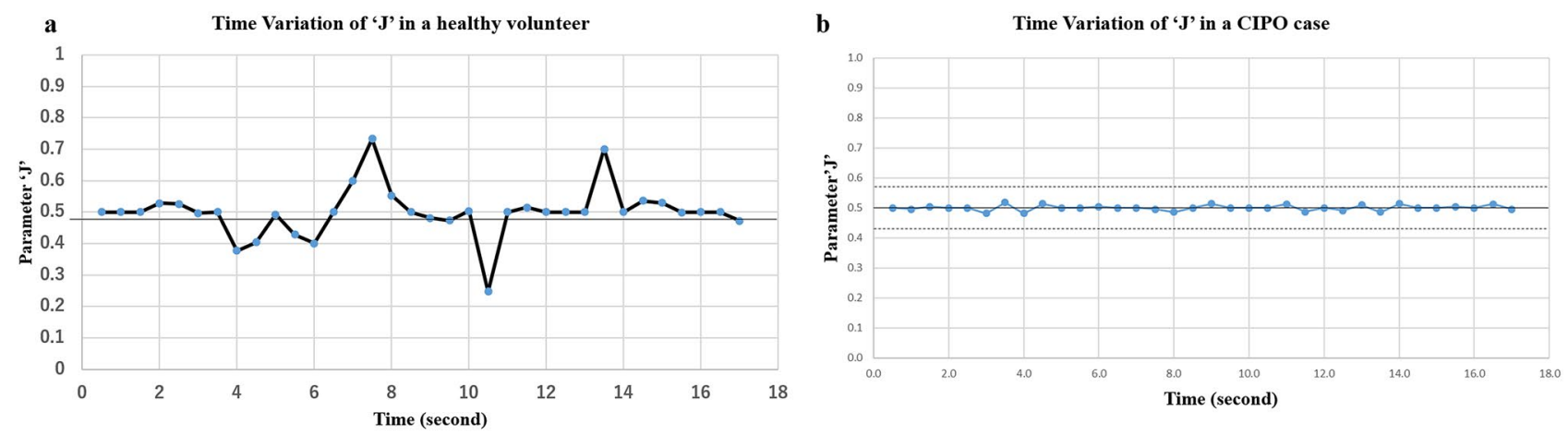

Figure 5. Time variation of parameter $\mathrm{J}$ in a healthy volunteer (a) and a case of chronic intestinal pseudoobstruction (b).

where $\mathrm{L}_{\mathrm{t}}$ is the luminal diameter at time $t$ and $\mathrm{L}_{\mathrm{t}+1}$ is that at time $\mathrm{t}+1$. The $\mathrm{J}$ values ranged from 0 to 1 . As the intestinal tract expands, the J value falls in the range of $0.5 \nsupseteq \mathrm{J}$ 1.0. Conversely, as the intestinal tract contracts, the value falls in the range of $0 \supsetneqq \mathrm{J} \lesseqgtr 0.5$. Moreover, $\mathrm{L}_{\mathrm{t}}=\mathrm{L}_{\mathrm{t}+1}$ leads to a J value of 0.5 . Therefore, this value indicates that the intestinal tract has no motility (Fig. 5). Thus, by using the relative index calculated from sequentially adjacent images, we made the most of the motion characteristic of the cine MRI.

Next, we defined two 34-dimensional vectors; one was a motility vector whose components were the values of $\mathrm{J}$, while the other was a non-motility vector whose components were all 0.5 . The magnitude of the intestinal tract motility was determined by the distance between the motility and non-motility vectors; as motility increases, the distance lengthens. Moreover, the mean of 35 luminal diameters was estimated. We adopted the Mahalanobis distance ${ }^{16}$ to obtain the invariance to the scaling of the coordinate axes.

The Mahalanobis distance $\mathrm{D}(\mathrm{m})$ between the mean $\mathrm{m}$ of 35 luminal diameters of the intestinal tract of a patient and the volunteer distribution of means from healthy volunteers was defined as follows:

$$
\mathrm{D}(m)=\frac{(m-\mu)^{2}}{\sigma^{2}}
$$

where $\mu$ and $\sigma^{2}$ are the sample mean and sample variance of the volunteer distribution, respectively. The sample mean and sample variance were estimated using the means for each of the intestinal tracts of healthy volunteers. Then, we calculated the logarithm of the Mahalanobis distance $\mathrm{D}(\mathrm{m})$; thus, any intestinal tract was represented as a two-dimensional vector whose components $\mathrm{x}_{1}$ and $\mathrm{x}_{2}$ were the logarithm of the Mahalanobis distance and distance variation per time, respectively. These $x_{1}$ and $x_{2}$ are new cine MRI parameters. In pattern recognition, the parameters $x_{1}$ and $x_{2}$ are called features. Each intestinal tract was represented as a feature vector in a twodimensional feature space.

Once the intestinal tract of the patient was selected, it was deemed to have impaired motility if $x_{1} \geq \alpha$ and $\mathrm{x}_{2} \leq \beta$, where $\alpha$ and $\beta$ are the threshold values (mentioned below), as shown in Fig. 6a. An intestinal tract that had no impairment in motility satisfied the condition $\mathrm{x}_{1}<\alpha$ or $\mathrm{x}_{2}>\beta$, which implied denial of the proposition $\mathrm{x}_{1} \geq \alpha$ and $\mathrm{x}_{2} \leq \beta$. The patient was deemed to have CIPO if any of the intestinal tracts was shown to have impaired motility, as shown in Fig. 6 b. 


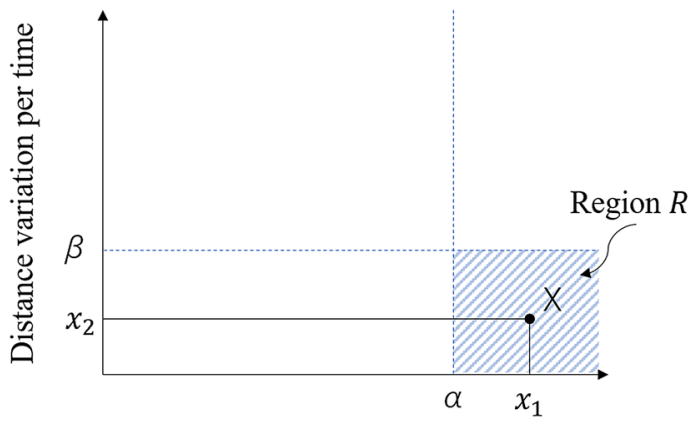

Logarithm of the Mahalanobis distance

$$
R=\left\{X=\left(x_{1}, x_{2}\right) \mid x_{1} \geqq \alpha \text { and } x_{2} \leqq \beta\right\}
$$

a: Explanation of the relationship between Region $R$ and Cut-off $(\alpha, \beta)$ in the feature space
Intestinal tracts of interest $\mathrm{X}_{1}, \mathrm{X}_{2}, \cdots, \mathrm{X}_{d}$ where $d$ is the number of intestinal tracts from a patient

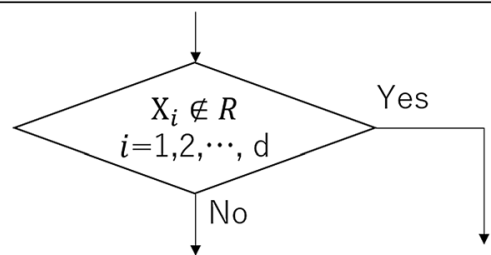

CIPO

Figure 6. (a) Scheme of assessment of intestinal motility. It is deemed to have impaired motility if $x_{1} \geq \alpha$ and $x_{2} \leq \beta$, where $\alpha$ and $\beta$ are the threshold values. An intestinal tract that had no impairment in motility satisfies the condition $x_{1}<\alpha$ or $x_{2}>\beta$, which implies denial of the proposition $x_{1} \geq \alpha$ and $x_{2} \leq \beta$. (b) The flowchart of cine-MRI diagnosis for chronic intestinal pseudo-obstruction (CIPO). A patient is deemed to have CIPO if any of the intestinal tracts with impaired motility was detected.

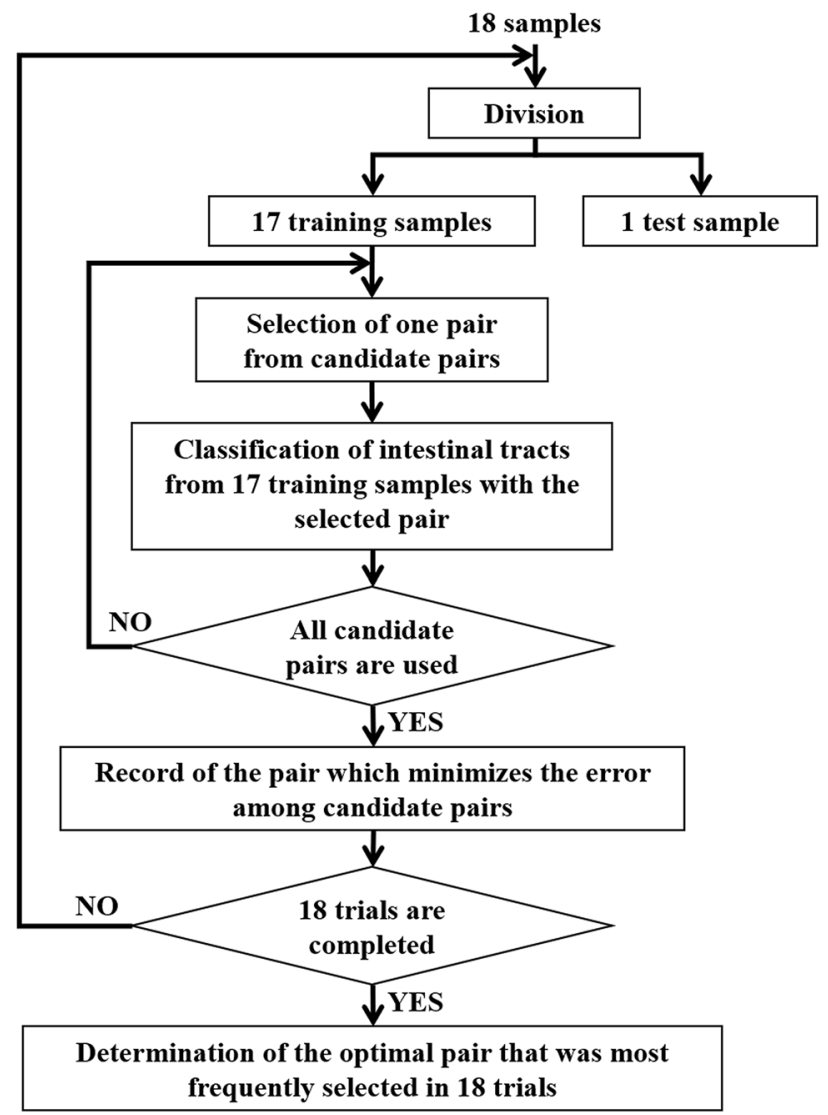

Figure 7. Scheme of optimization of threshold values.

As the values of $\alpha$ and $\beta$ are critical for classifying individuals, we determined the optimal $\alpha$ and $\beta$ pairing (i.e., that which minimized error) from among candidate pairs (Fig. 7). To determine the most robust pair that optimally takes into account the variability of samples, we used the leave-one-out method ${ }^{17}$, which generates artificial variability among the patients with the use of resampling ${ }^{18}$. There were 18 available samples; according to the leave-one-out method, one sample was excluded, and the remaining 17 were used as training samples. Note that the test sample was not used in optimization. Next, an $\alpha$ and $\beta$ pair was selected from candidate pairs. 


\begin{tabular}{|l|l|l|l|l|l|l|l|l|}
\hline & & & & & & \multicolumn{2}{l|}{ Cine MRI } & \\
Case No & Age $(\mathbf{y})$ & Sex & BMI $\left(\mathbf{k g} / \mathbf{m}^{2}\right)$ & CIPO duration (months) & Symptoms $^{\mathbf{a}}$ & $\mathbf{X}_{\mathbf{1}}$ & $\mathbf{X}_{2}$ & Treatment \\
\hline 1 & 37 & M & 18.2 & 180.3 & D, V & 1.79 & 0.22 & Medication \\
\hline 2 & 52 & M & 19.4 & 35.7 & D, V, P & 2.68 & 0.10 & Medication, Surgical bypass, HPN \\
\hline 3 & 61 & M & 17.1 & 6.5 & D, V & 1.12 & 0.16 & Medication \\
\hline 4 & 55 & F & 18.2 & 81.0 & D, V & 2.79 & 0.04 & Medication, surgical bypass, HPN \\
\hline 5 & 35 & F & 11.1 & 37.6 & D, P, C & 2.01 & 0.10 & Surgical bypass \\
\hline 6 & 54 & F & 15 & 40.1 & D, P & 2.33 & 0.13 & Surgical bypass \\
\hline 7 & 40 & F & 17.3 & 156.6 & D, C & 4.26 & 0.04 & Medication \\
\hline
\end{tabular}

Table 1. Clinical course and cine magnetic resonance imaging findings in patients with chronic intestinal obstruction. BMI, body mass index; CIPO, chronic intestinal obstruction; HPN, home parenteral nutrition; MRI, magnetic resonance imaging. ${ }^{a} \mathrm{D}$ : abdominal distention, V: vomiting, P: Abdominal pain, C: constipation. $\mathrm{x}_{1}$ : the logarithm of the Mahalanobis distance, $\mathrm{x}_{2}$ : the distance variation per time.

Thereafter, we determined whether each intestinal tract of a training sample had severe impairment of motility using the selected $\alpha$ and $\beta$ pair. If $x_{1} \geq \alpha$ and $x_{2} \leq \beta$, the intestinal tract was classified as an impaired one, and the number of misclassified intestinal tracts was counted from among intestinal tracts of 17 training samples to estimate the error. This was conducted for each candidate pair. Ultimately, we selected the pair that produced the lowest error among the intestinal tracts of 17 training samples. The above operation was repeated until each of the 18 samples had been excluded once. We determined the optimal pair that was most frequently selected from among the 18 trials.

Statistics. Continuous variables, such as patient age and body mass index are presented as medians and interquartile ranges, while categorical variables such as sex are expressed as numbers and percentages. All statistical analyses were performed using EZR (Saitama Medical Center, Jichi Medical University, Saitama, Japan), which is a graphical user interface for R (The R Foundation for Statistical Computing, Vienna, Austria). More precisely, it is a modified version of $\mathrm{R}$ commander designed to add statistical functions frequently used in biostatistics $^{19}$.

\section{Results}

Seven patients with CIPO, including three men (42.9\%) and four women (57.1\%), whose age was 52 (38.5-54.5) years underwent cine MRI; their characteristics are shown in Table 1. The disease duration was $40.1(36.6-118.8)$ months, and all the patients were underweight (body mass index $=17.3[16.2-18.2] \mathrm{kg} / \mathrm{m}^{2}$ ) and had abdominal distention. Cine MRI examination was also performed on 11 healthy volunteers comprising six men (54.5\%) and five women $(45.5 \%)$ with an age of $35(29.0-41.5)$ years.

Threshold value for separating patients with CIPO from healthy volunteers. The logarithm of the Mahalanobis distance, as well as the distance variation per time, were calculated for each of the intestinal tracts from both patients with CIPO and healthy volunteers, and the data are shown graphically in Fig. 8 (Supplementary File S1).

The optimal pair of $\alpha=1.10$ and $\beta=0.15$ was considered to be robust for the variability of samples. The parameters with these values produced a sensitivity of $1.00(7 / 7)$ and specificity of $0.82(9 / 11)$ (Fig. 9a); the error was $0.11(2 / 18)$. On the other hand, a specificity of $1.00(11 / 11)$ and sensitivity of $0.86(6 / 7)$ were obtained when values of $\alpha=2.00$ and $\beta=0.15$ were used (Fig. $9 b$ ).

Severity of CIPO and cine MRI findings. The severity of CIPO was determined based on the treatment required: mild CIPO cases were controlled by medication only; moderate cases required surgical intervention; and severe cases required permanent HPN (Table 1). The mean vector of the intestinal tract from each case was estimated as a representative characteristic. Table 1 shows the two components of the mean vector. Any of the intestinal tracts of severe cases belonged to the region defined by $\mathrm{x}_{1} \geq 1.10$ and $\mathrm{x}_{2} \leq 0.15$ in the two-dimensional feature space. Furthermore, in patients with CIPO, Pearson's analysis of the logarithm of the Mahalanobis distance and the distance variation per time revealed a mild inverse correlation of -0.52 (Fig. 10). When analyzing all participants, including patients and volunteers $(n=18)$ as well as volunteers alone $(n=11)$, no correlation was found (the Pearson's correlation coefficients were -0.15 and 0.01 , respectively).

\section{Discussion}

We successfully established cine MRI parameters that could different iate patients with CIPO from healthy volunteers. Our analysis also revealed that all patients with CIPO had impaired small intestinal motility. We assumed that for the intestinal tracts from a patient with CIPO, $\mathrm{x}_{2}$ became small, with the logarithm of the Mahalanobis distance $\mathrm{x}_{1}$ large. The results showed that the negative correlation between $\mathrm{x}_{1}$ and $\mathrm{x}_{2}$ was observed only for patients with CIPO.

We used the original parameters 'logarithm of the Mahalanobis distance' and 'distance variation per time' in this study because other previously reported parameters, such as intestinal 'amplitude' and 'contraction cycle' 


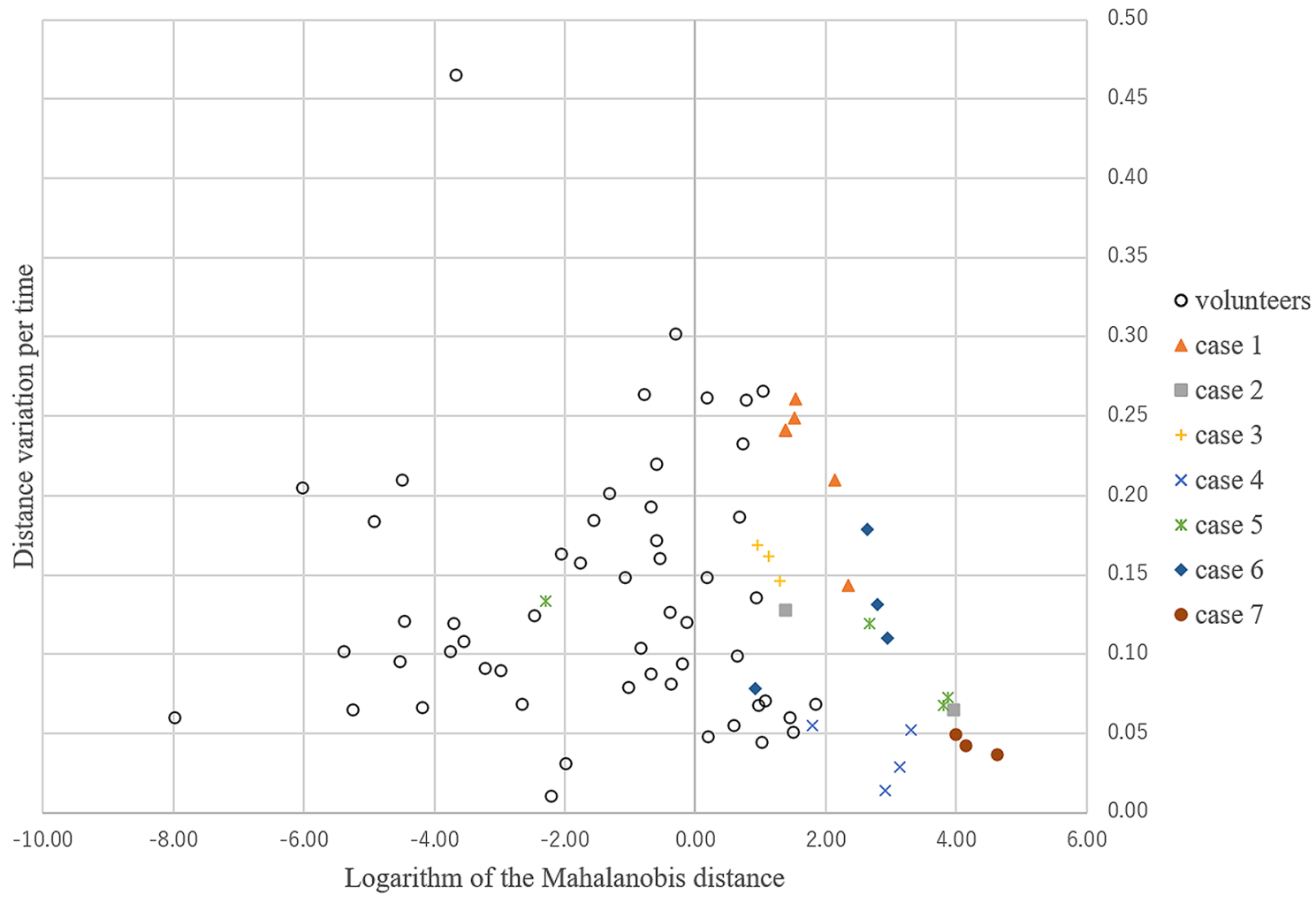

Figure 8. Scatter diagram of the logarithm of the Mahalanobis distance (horizontal axis) and distance variation per time (vertical axis) in patients with chronic intestinal pseudo-obstruction and healthy volunteers.

were difficult to determine during our previous efforts. This was presumably because of the various types of small intestinal contraction that occur concurrently, including peristalsis, segmentation, and phase III migrating motor complex ${ }^{8,20}$. This makes it difficult to distinguish the type of each contraction definitively; moreover, the 'amplitude' and 'contraction cycle' are not considered single or regular. Instead, in this study, bowel contraction or expansion was evaluated by $\mathrm{J}<0.5$ or $\mathrm{J}>0.5$, which just focus on the rate of variability per time. Given that luminal diameters of patients with CIPO have diagnostic significance only when they are compared with those of healthy controls, the Mahalanobis distance was thought to be a proper metric in evaluating luminal diameters, since it normalized the luminal diameter of patients with CIPO using that of healthy volunteers. Furthermore, the other advantage of using the Mahalanobis distance is that it is not affected by the scan resolutions and can be applied to scans from different types of MRI equipment, which is different from the Euclidean distance (Supplementary File S2).

Although the feature vectors of healthy volunteers had widely distributed values in this study, the threshold value of 1.10 for the parameter $\mathrm{x}_{1}$ was found to be satisfactory for CIPO diagnosis. Furthermore, the luminal diameters were selected for analysis from all parts of the small intestine because we could not always measure these diameters in specific areas (i.e., the proximal jejunum, middle intestine, and distal ileum) given that the analyses were performed on sliced views, wherein certain parts of the small intestine were often in a blind spot and thus invisible. Another drawback was that the intestines sliced horizontally or perpendicularly were not always captured given the small intestine's tortuousness in the three-dimensional abdominal cavity.

A key finding in this study was that all patients with CIPO had impaired small intestinal motility. However, in some cases, lower colonic propulsion may have been impaired, which further inhibited small bowel propagation. Furthermore, the severity of CIPO was reflected in the cine MRI findings. Therefore, our calculated parameters may potentially be useful for clinical decision-making. In contrast, the parameters calculated for healthy volunteers had a wide range, which was a notable observation. Further studies of cine MRI are required to reliably differentiate CIPO from other disorders, such as irritable bowel syndrome, although determining the definitive parameters is likely to be challenging. At a minimum, longer cine MRI observation is necessary, and the extent of small intestinal contraction (peristalsis, segmentation, and migrating motor complex), should be analyzed.

Based on our results, using cine MRI together with computer-assisted or artificial intelligence-driven diagnosis of gastrointestinal motility may have potential to accurately identify patients with CIPO, although several drawbacks should be addressed (such as the limitations of sliced-based analysis). Although there was a bias in the selection of the intestinal tract and positioning of the luminal diameter, we adopted the effective method of visually measuring only one measurement point, i.e., one luminal diameter for each of the corresponding intestinal tracts. Our study focused on small intestinal motility, and colonic motility should be further explored in additional investigations. The small intestine usually functions to move and clear its luminal contents before more food is ingested. In contrast, the colon is idle up to $90 \%$ of the time. Hence, cine MRI may be unsuitable for the evaluation of colonic movement given its glacial pace. 

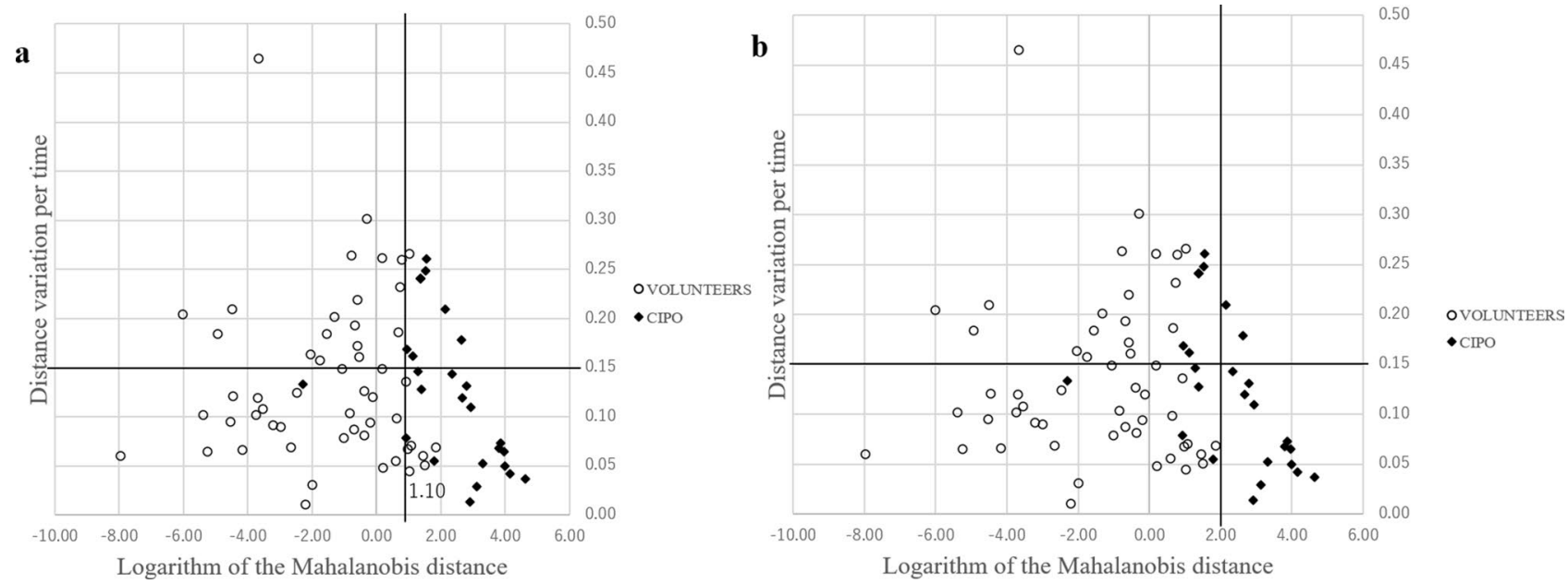

Figure 9. Scatter diagram for determining sensitivity and specificity in two-dimensional feature space. (a) $\alpha=1.10$ and $\beta=0.15$ can be used to obtain the best sensitivity for chronic intestinal pseudo-obstruction (CIPO) diagnosis. (b) $\alpha=2.00$ and $\beta=0.15$ can be used to obtain the maximum specificity for CIPO diagnosis.

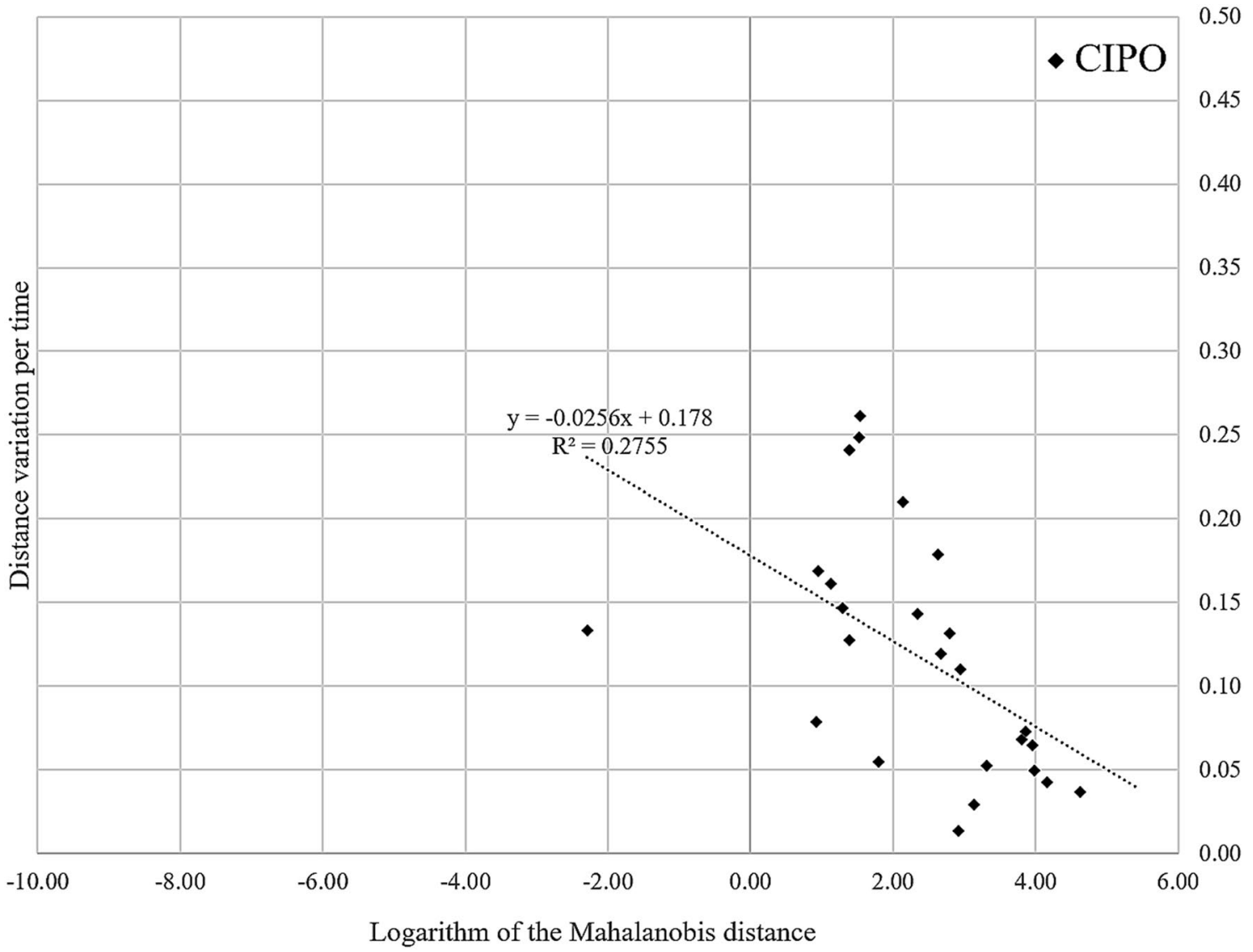

Figure 10. In patients with chronic intestinal obstruction, the logarithm of the Mahalanobis distance and distance variation per time were inversely correlated.

Our study had several limitations that should be acknowledged. First, only a small number of patients with CIPO were analyzed because of the rarity of this disorder. Therefore, data analysis between CIPO and healthy volunteers was limited. Classification experiments were conducted using the $\alpha$ and $\beta$ values optimized by the leave-one-out method with 18 samples. The performance should be evaluated for independent test samples. However, this remains one of the issues to be studied further. Second, we classified the severity of CIPO based on the required treatment; however, severity is determined using a complicated scale in real-world clinical settings. For example, patient 7 had severe symptoms that were controlled with many medications and well-balanced 
meals. Third, the backgrounds of patients with CIPO and healthy volunteers, such as age, sex, and body mass index were different, which may also have affected intestinal motility.

In conclusion, our new cine MRI parameters have the potential to assist in the differential diagnosis of patients suspected of having CIPO, including the severity thereof. The wide ranges of distributions for feature vectors of healthy volunteers may reflect the complicated function of the small intestine, which should be elucidated using additional cine MRI studies in the future.

\section{Data availability}

All data used in the study are already provided in the tables, figures, and online supplementary materials.

Received: 4 June 2021; Accepted: 12 November 2021

Published online: 26 November 2021

\section{References}

1. Ohkubo, H. et al. An epidemiologic survey of chronic intestinal pseudo-obstruction and evaluation of the newly proposed diagnostic criteria. Digestion 86, 12-19 (2012).

2. Amiot, A. et al. Long-term outcome of chronic intestinal pseudo-obstruction adult patients requiring home parenteral nutrition. Am. J. Gastroenterol. 104, 1262-1270 (2009).

3. De Giorgio, R., Sarnelli, G., Corinaldesi, R. \& Stanghellini, V. Advances in our understanding of the pathology of chronic intestinal pseudo-obstruction. Gut 53, 1549-1552 (2004).

4. Knowles, C. H., Lindberg, G., Panza, E. \& De Giorgio, R. New perspectives in the diagnosis and management of enteric neuropathies. Nat. Rev. Gastroenterol. Hepatol. 10, 206-218 (2013).

5. Di Nardo, G. et al. Review article: Molecular, pathological and therapeutic features of human enteric neuropathies. Aliment. Pharmacol. Ther. 28, 25-42 (2008).

6. Sato, H. et al. Esophageal high-resolution manometry for diagnosing the severity of the chronic intestinal pseudo-obstruction: A case series. Dig. Dis. Sci. https://doi.org/10.1007/s10620-020-06701-9 (2020).

7. Camilleri, M. \& Linden, D. R. Measurement of gastrointestinal and colonic motor functions in humans and animals. Cell Mol. Gastroenterol. Hepatol. 2, 412-428 (2016).

8. Malagelada, C. \& Malagelada, J. R. Small bowel motility. Curr. Gastroenterol. Rep. 19, 26 (2017).

9. Keller, J. et al. Expert consensus document: Advances in the diagnosis and classification of gastric and intestinal motility disorders. Nat. Rev. Gastroenterol. Hepatol. 15, 291-308 (2018).

10. Tominaga, K. et al. Variation in small bowel transit time on capsule endoscopy. Ann. Transl. Med. 8 (2020),

11. Arnold, J. R. \& McCann, G. P. Cardiovascular magnetic resonance: Applications and practical considerations for the general cardiologist. Heart 106, 174-181 (2020).

12. Ohkubo, H. et al. Assessment of small bowel motility in patients with chronic intestinal pseudo-obstruction using cine-MRI. Am. J. Gastroenterol. 108, 1130-1139 (2013).

13. Fuyuki, A. et al. Clinical importance of cine-MRI assessment of small bowel motility in patients with chronic intestinal pseudoobstruction: A retrospective study of 33 patients. J. Gastroenterol. https://doi.org/10.1007/s00535-016-1251-8 (2016).

14. Jonge, C. S. de, de Jonge, C. S., Smout, A. J. P., Nederveen, A. J. \& Stoker, J. Evaluation of gastrointestinal motility with MRI: Advances, challenges and opportunities. Neurogastroenterol. Motil. 30, e13257 (2018).

15. Jain, A. K. Fundamentals of Digital Image Processing (Prentice Hall, Prentice, 1989).

16. Mahalanobis, P. C. On the generalized distance in statistics. Proc. Natl. Inst. Sci. India 12, 49-55 (1936).

17. Lachenbruch, P. A. Estimation of Error Rates in Discriminant Analysis (University of California at Los Angeles, 1965).

18. Ogihara, H., Iizuka, N. \& Hamamoto, Y. Prediction of early recurrence of liver cancer by a novel discrete Bayes decision rule for personalized medicine. Biomed. Res. Int. 2016, 8567479 (2016).

19. Kanda, Y. Investigation of the freely available easy-to-use software 'EZR' for medical statistics. Bone Marrow Transplant. 48, 452-458 (2013).

20. Kumral, D. \& Zfass, A. M. Gut movements: A review of the physiology of gastrointestinal transit. Dig. Dis. Sci. 63, 2500-2506 (2018).

\section{Acknowledgements}

We would like to thank Editage [http://www.editage.com] for editing and reviewing this manuscript for English language.

\section{Author contributions}

H.S. wrote the main manuscript text and performed volunteers' recruitment. H.O. and Y.H. analyzed data. K.T., Y.K., Y.K., K.T., and J.Y. recruited cases. S.T. supervised the study. All authors reviewed the manuscript.

\section{Funding}

This work was supported by JSPS Grants-in-Aid for Scientific Research: Grant Nos. 18K15805 and 20 K08279.

\section{Competing interests}

The authors declare no competing interests.

\section{Additional information}

Supplementary Information The online version contains supplementary material available at https://doi.org/ 10.1038/s41598-021-02268-1.

Correspondence and requests for materials should be addressed to H.S.

Reprints and permissions information is available at www.nature.com/reprints.

Publisher's note Springer Nature remains neutral with regard to jurisdictional claims in published maps and institutional affiliations. 
(c) (i) Open Access This article is licensed under a Creative Commons Attribution 4.0 International cc) License, which permits use, sharing, adaptation, distribution and reproduction in any medium or format, as long as you give appropriate credit to the original author(s) and the source, provide a link to the Creative Commons licence, and indicate if changes were made. The images or other third party material in this article are included in the article's Creative Commons licence, unless indicated otherwise in a credit line to the material. If material is not included in the article's Creative Commons licence and your intended use is not permitted by statutory regulation or exceeds the permitted use, you will need to obtain permission directly from the copyright holder. To view a copy of this licence, visit http://creativecommons.org/licenses/by/4.0/.

(C) The Author(s) 2021 